\title{
Sílidos (Syllidae: Polychaeta) del Parque Nacional de Coiba (Pacífico, Panamá)
}

\author{
María Teresa Aguado ${ }^{1,2}$ \& Guillermo San Martín ${ }^{1,3}$ \\ 1 Laboratorio de Biología Marina e Invertebrados, Departamento de Biología (Zoología), Facultad de Ciencias, \\ Universidad Autónoma de Madrid, Cantoblanco, 28049 Madrid, España \\ 2 maite.aguado@uam.es \\ 3 guillermo.sanmartin@uam.es
}

Recibido 18-IV-2002. C Corregido 26-X-2004. Aceptado 15-III-2006.

\begin{abstract}
Syllids (Syllidae: Polychaeta) from Coiba National Park (Pacific of Panama). During a study carried out on soft bottoms from Coiba National Park (Panama), 218 specimens of syllids (Annelida: Polychaeta) belonging to 19 interstitial species have been identified. Two species are new reports for the Pacific Ocean, Exogone (Exogone) arenosa Perkins, 1981 and Streptosyllis websteri Southern, 1914; five are new for Panama, E. (E.) dispar (Webster, 1879); E. (E.) longicornis Westheide, 1974; Salvatoria mediodentata (Westheide, 1974); Pionosyllis heterocirrata (Hartmann-Schröder, 1959) and Syllis glarearia (Westheide, 1974). A characteristic not mentioned in the original description is herein reported for E. (E.) longicornis: the presence of triangular subterminal processes in the spiniger-like compound chaetae of chaetiger one. Specimens of the species Syllis botosaneanui Hartmann-Schröder, 1973 harboring inside embryos of various stages of development have been found; this is the first report for this species as viviparous. Lastly, one specimen of the genus Syllis has been found that is mainly characterized by its long pharynx, two dorsal prostomial lobes, and compound chaetae with short blades and long spinulation. Because we consider that one specimen is not sufficient to describe a new species we refer it as Syllis sp. Rev. Biol. Trop. 54 (3): 725-743. Epub 2006 Sept. 29.
\end{abstract}

Key words: polychaeta, Syllidae, Coiba, Pacific, Panama, viviparity.

El Parque Nacional de Coiba es uno de los parques nacionales con mayor superficie marina protegida del mundo (216.543 ha). Se encuentra situado a 150 millas náuticas de la costa Pacífica de Panamá, y está formado por un archipiélago; la mayor de sus islas es la que le da nombre al Parque. La descripción del medio marino del Parque está recogida en López et al. (1997, 2002), San Martín (2000, 2001) y San Martín et al. (1997a, b).

Como resultado de las expediciones llevadas a cabo desde 1996 por el equipo del laboratorio de Biología Marina e Invertebrados de la Universidad Autónoma de Madrid, la criptofauna coralina del Parque y, en particular, la fauna de poliquetos han sido estudiados en varios trabajos (San Martín et al. 1997a, b,
Capa et al. 2001a, b, c). Los tres trabajos últimos se refieren específicamente a la familia Syllidae de la criptofauna coralina del Parque. Es por ello que el presente trabajo se ha centrado en las especies de sílidos identificados en muestras de arena, lo cual permite conseguir un inventario más completo de esta familia en Coiba.

Algunos trabajos anteriores a 1997 en los que se estudian los poliquetos del Pacífico panameño entre ellos, la familia Syllidae pueden consultarse en Capa et al. (2001a, b, c).

\section{MATERIALES Y MÉTODOS}

El material procede de cinco muestras tomadas en sustratos blandos en tres estaciones 
del Parque Nacional de Coiba durante el mes de Septiembre de 1998. Las muestras tomadas en Mali Rock ( $7^{\circ} 39^{\prime} \mathrm{N}, 81^{\circ} 41^{\prime} 40^{\prime \prime}$ W) y Granito de Oro ( $7^{\circ} 35^{\prime} 30^{\prime \prime}$ N, 81 ${ }^{\circ} 42^{\prime} 30^{\prime \prime}$ W) son de arenas gruesas submareales, procedentes de la destrucción de coral (a 10 m y 0.5 m de profundidad, respectivamente). Las tres restantes son intermareales, de arena media a gruesa y fueron tomadas en la playa de El Gambute

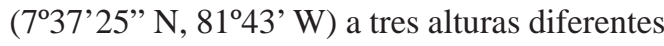
(nivel de pleamar, nivel intermedio de marea y nivel de bajamar).

Las muestras se obtuvieron introduciendo en el sedimento un tubo de PVC de 1 litro de capacidad. Posteriormente fueron filtradas utilizando un tamiz con luz de malla de $0.1 \mathrm{~mm}$ de diámetro, fijadas en formol (10\%) y preservadas en etanol (70\%) tras la identificación de los ejemplares. Para la identificación se ha utilizado una lupa binocular Olympus SZ30, un microscopio óptico Olympus CH30 y un microscopio óptico Nikon 104 provisto del sistema de contraste de fases interferencial de Nomarsky. Para la realización de los dibujos se ha empleado una cámara clara Nikon y un ocular milimetrado. Las fotografías al microscopio electrónico de barrido (MEB) se llevaron a cabo en el SIDI (Servicio Interdepartamental de Investigación) de la Universidad Autónoma de Madrid. Los ejemplares están depositados en el Museo Nacional de Ciencias Naturales de Madrid y en la colección de Poliquetos del Laboratorio de Biología Marina e Invertebrados de la Universidad Autónoma de Madrid.

\section{RESULTADOS}

Han sido identificados 218 ejemplares de sílidos, pertenecientes a nueve géneros y 19 especies, de las cuales dos son nuevas citas para el Pacífico: Exogone arenosa Perkins, 1981 y Streptosyllis websteri Southern, 1914. Otras cinco son nuevas citas para Panamá: E. dispar (Webster, 1879); E. longicornis Westheide, 1974; Salvatoria mediodentata (Westheide, 1974); Pionosyllis heterocirrata (Hartmann-
Schröder, 1959) y Syllis glarearia (Westheide, 1974). Para E. longicornis se detalla una nueva característica no mencionada en descripciones previas, la posesión de proyecciones terminales en los mangos de las pseudoespinígeras del primer setígero. Se describe la especie Syllis botosaneaunui Hartmann-Schröder, 1973 como especie vivípara $\mathrm{y}$, por último, se describe la especie Syllis sp., que probablemente sea una especie no descrita hasta el momento, pero al tener un sólo ejemplar preferimos no darle un nuevo nombre.

\section{Subfamilia Exogoninae \\ Langerhans, 1879 \\ Género Brania \\ Quatrefages, 1866 \\ Brania sp.}

Material examinado: Granito de Oro (un ejemplar).

Discusión: El ejemplar está incompleto y en mal estado de conservación, por lo que no puede ser determinado hasta nivel de especie.

$$
\begin{aligned}
& \text { Género Exogone } \\
& \text { Örsted, } 1845 \\
& \text { Subgénero Exogone } \\
& \text { Örsted, 1845 } \\
& \text { E. (Exogone) arenosa } \\
& \text { Perkins, } 1981
\end{aligned}
$$

E. arenosa: Perkins, 1981: 1094-107, Fig. 5 g-j, 6. E. (Exogone) arenosa: San Martín, 1991: 736.

Material examinado: Granito de Oro (50 ejemplares).

Discusión: E. arenosa, E. lourei Berkeley \& Berkeley, 1938 y E. pseudolourei San Martín, 1991 son especies muy similares, todas ellas presentes en el Caribe, y cuyas sedas compuestas son muy parecidas, pues siempre presentan una notoria expansión triangular en la región subterminal del mango. E. arenosa se caracteriza por tener un proventrículo largo (30 filas de células musculares, siete segmentos) y una espina en la punta de las sedas 
simples dorsales; sin embargo, E. lourei y E. pseudolourei tienen un proventrículo corto (aproximadamente 20 filas de células musculares, 2.5 segmentos) y no presentan espina en las sedas simples dorsales. E. pseudolourei se diferencia de $E$. lourei en la forma y grosor de la seda simple dorsal.

Perkins (1981) y San Martín (1991) diferencian estas especies principalmente por la longitud del proventrículo. San Martín (1991) describe ejemplares de Cuba de E. arenosa con y sin espina en la seda simple dorsal y siendo ésta, cuando existe, muy variable en tamaño. Atendiendo a este criterio, los ejemplares de Coiba poseen todos ellos un proventrículo largo por lo que son considerados como E. arenosa, aunque algunos presentan una espina más reducida que la descrita por Perkins (1981), y algunos carecen de ella.

Distribución: Atlántico (Florida, Cuba, islas Canarias) Pacífico (Panamá, primera cita).

\section{Exogone (Exogone) breviantennata} Hartmann-Schröder, 1959

E. breviantennata: Hartmann-Schröder 1959: 125, Fig. 75-78; Zottoli \& Long 2000: 502, Fig. 1-5.

E. (Exogone) breviantennata: San Martín 1991: 730; 2005: 141-142, Fig. 81 e, 89 a-i; Núñez et al. 1992: 47, Fig. 3; López et al. 1997: 63.

Material examinado: Granito de Oro (seis ejemplares).

Distribución: Pacífico (Canadá, México, Ecuador, Galápagos, Japón, Australia y Panamá) Atlántico (Venezuela, Cuba, Panamá y Canarias) Índico (Australia).

\section{Exogone (Exogone) dispar}

(Webster, 1879)

E. dispar: Westheide 1974: 106, Fig. 48 a-h, 49 a-d; Perkins 1981: 1090; Uebelacker 1984: 43, Fig. 30-36.

E. (Exogone) dispar: San Martín 1991: 729; 2005: 137-138, Fig. 81 f, 85 a-g.
Material examinado: Granito de Oro (tres ejemplares).

Distribución: Mediterráneo Atlántico (Norte América, Golfo de México, Cuba) Pacífico (Galápagos, Australia y Panamá, primera cita).

\section{E. (Exogone) longicornis}

Westheide, 1974, Fig. 1

E. longicornis: Westheide, 1974: 309-313, Fig. 54-55.

Material examinado: Granito de Oro (13 ejemplares).

Material adicional: Dos ejemplares de Galápagos: Serie tipo, Westheide, 1974. Dos ejemplares de Australia: Isla de Beacon, $113^{\circ} 47^{\prime}$ E 28 25.5' S, coral muerto, -12 m (un ejemplar) (AM W26666); Isla de Wallaby, $113^{\circ} 40.9^{\prime} \mathrm{E}$ 28 27.9' S en Posidonia australis, $-2 \mathrm{~m}$ (un ejemplar) (AM W26667).

Descripción: Ejemplar de mayor tamaño de 33 setígeros, $2.9 \mathrm{~mm}$ de longitud y $0.2 \mathrm{~mm}$ de anchura, a nivel del proventrículo. Prostomio semicircular con dos pares de ojos dispuestos en trapecio, el par anterior bilobulado. Tres antenas insertas entre el par anterior de ojos, la central considerablemente más larga, cinco a seis veces más que las laterales, estrechándose distalmente, llegando a parecer articulada. Palpos robustos, más largos que el prostomio. Segmento bucal tapado parcialmente por el prostomio, provisto de un par de cirros tentaculares ovoides, de menor tamaño que el resto de los cirros dorsales, y cirros ventrales ovalados, ligeramente más pequeños que los dorsales (Fig. 1A). Pseudoespinígera del primer setígero con el mango engrosado en su parte distal, con prolongación subtriangular provista en su cara superior de espinas muy finas; artejo de $13.2 \mu \mathrm{m}$ de longitud, finamente espinulado y con el extremo bífido (Fig. 1B). En el primer setígero, aparecen también cinco sedas falcígeras de mango densamente espinulado y con artejos, de $4.6 \mu \mathrm{m}$ de longitud, provistos de un gran diente secundario y un diente principal mucho más corto y fino que el 

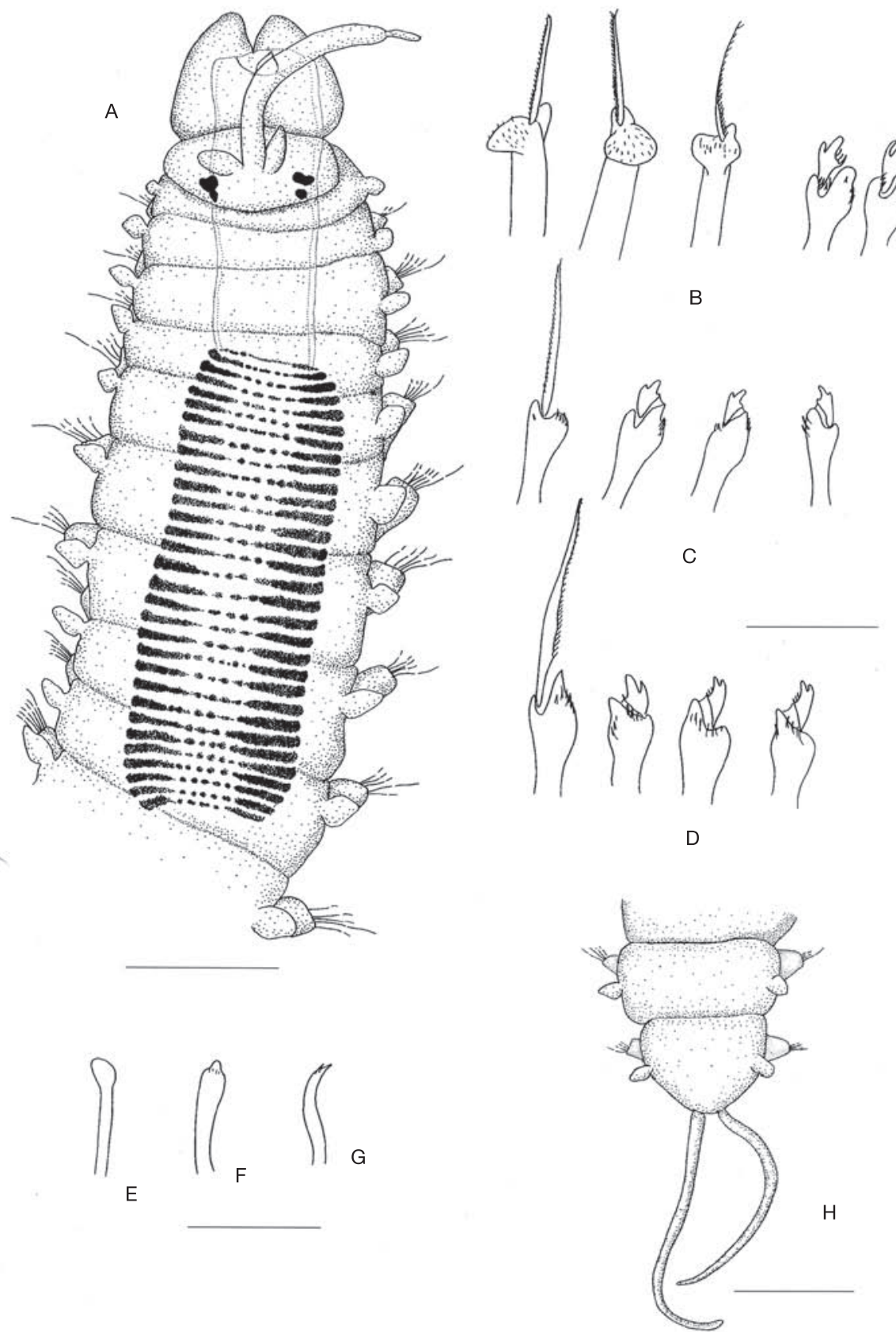

Fig. 1. Exogone (Exogone) longicornis. A. Parte anterior, vista dorsal; B. Sedas compuestas, primer setígero; C. Sedas compuestas, setígero medio; D. Sedas compuestas, setígero posterior; E. Acícula, setígero medio; F. Seda simple dorsal, setígero posterior; G. Seda simple ventral, setígero posterior; H. Pigidio, vista dorsal. Escala A, H. 0.16 mm; B-G. $20 \mu \mathrm{m}$. B-E. (modificado de San Martín 2005).

Fig. 1. Exogone (Exogone) longicornis. A. Anterior part, dorsal view; B. Compound chaetae, first chaetiger; C. Compound chaetae, medium chaetiger; D. Compound chaetae, posterior chaetiger; E. Acicula, médium chaetiger; F. Dorsal, simple chaeta, posterior chaetiger; G. Ventral, simple chaeta, posterior chaetiger; H. Pygidium, dorsal view. Scale A, H. 0.16 mm; B-G. 20 m. B-E. (after San Martín 2005). 
anterior y 2-3 espinas largas y delgadas (Fig. 1B). Siguientes segmentos, con el mango de la pseudoespinígera no engrosado en ninguno de los casos. Segundo setígero con una seda pseudoespinígera ligeramente bífida, carácter que se va perdiendo en los siguientes setígeros. Pseudospinígeras de los segmentos anteriores de mayor longitud que la del primer setígero (29.7 $\mu \mathrm{m})$ y 5-6 falcígeras similares a las del primer setígero, pero con espinas más cortas (Fig. 1C). Las pseudoespinígeras decrecen en longitud en los segmentos posteriores (14.5 $\mu \mathrm{m})$ y en las falcígeras no se observan las espinas. Seda dorsal simple con un engrosamiento espinulado subdistal, a partir del primer setígero. Seda simple ventral, en los segmentos posteriores, curvada y bidentada con el diente secundario más desarrollado que el principal (Fig. 1G). Parápodos con una sola acícula acabada en un engrosamiento (Fig. 1E). Pigidio con un par de cirros anales largos (Fig. 1H). Faringe larga que ocupa, aproximadamente cinco setígeros, con un grueso diente en su parte anterior. Proventrículo largo de unas 30 filas de células musculares, llegando a ocupar cuatro o cinco setígeros (Fig. 1A). Varios ejemplares tienen la parte posterior cargada de huevos, en el interior de los segmentos comprendidos entre el 14 y el 23. También se ha encontrado un ejemplar con un embrión lateral.

Discusión: Han sido revisados dos ejemplares de Galápagos pertenecientes a la serie tipo que, al igual que en los ejemplares de Coiba y otros procedentes de Australia, poseen los mangos de la pseudoespinígeras del primer setígero modificados con procesos triangulares subterminales, característica que no se recoge en la descripción original.

Distribución: Pacífico (Islas Galápagos, Australia y Panamá, primera cita).

\section{Exogone (Exogone) lourei \\ Berkeley \& Berkeley, 1938}

E. (Exogone) lourei: Berkeley \& Berkeley, 1938: 44-47, Fig. 6-10; Banse 1972: 200, Fig. 5 a-d; Perkins 1981: 1092; Uebelacker 1984:
39-41, Fig. 33-34 (en parte); San Martín 1991: 735; 2005: 129-130, Fig. 78 a-j; Núñez et al. 1992: 46-47; Kudenov \& Harris 1995: 15-17, Fig. 1.3; Capa et al. 2001c: 623.

Material examinado: Mali Rock (2 ejemplares).

Material adicional: Material de fondos duros de Coiba (T2F97).

Discusión: Los ejemplares coinciden con la descripción original y con el material revisado de fondos duros de Coiba (Capa et al. 2001c).

Distribución: Pacífico (desde Canadá hasta Panamá), Atlántico (Islas de Cabo Verde y Canarias), Caribe (Golfo de México y Cuba), Índico (Australia).

\section{Género Salvatoria \\ Mc Intosh, 1885}

Siguiendo los criterios de San Martín (2005), las especies descritas o citadas dentro del género Grubeosyllis Verrill, 1900, pasan a ser incluidas en el género Salvatoria. Las dos especies de este género encontradas en este trabajo constituyen una nueva combinación.

\section{Salvatoria heterocirra}

(Rioja,1941), n. comb.

Brania heterocirra: Rioja, 1941: 700, pl. 3, Fig. 11-13; Westheide 1974: 83, Fig. 38, 39.

Grubeosyllis heterocirra: López et al. 1997: 63; Capa et al. 2001c: 624.

Material examinado: Granito de Oro (dos ejemplares).

Distribución: Pacífico (Islas Galápagos, México y Panamá), Caribe (Cuba).

\section{Salvatoria mediodentata}

(Westheide, 1974), n. comb

Brania mediodentata: Westheide, 1974: 93-94, Fig. 42 a-c, 43; Russell 1991: 52-54, Fig. 1.

Grubeosyllis mediodentata: Díaz-Castañeda \& San Martín 2001: 710-712, Fig. 2-4. 
Material examinado: Granito de Oro (29 ejemplares).

Distribución: Caribe (Cuba, Belice) Pacífico (Islas Galápagos, México y Panamá, nueva cita).

$$
\begin{aligned}
& \text { Subfamilia Eusyllinae } \\
& \text { Malaquin, } 1893 \\
& \text { Género Odontosyllis } \\
& \text { Claparède, } 1963 \\
& \text { Odontosyllis fulgurans }
\end{aligned}
$$

Audouin \& Milne Edward, 1834

O. fulgurans: Pettibone 1963: 122, Fig. 35 c; Imajima \& Hartman 1964: 113; San Martín 1984: 93-97, Fig. 14; 2003: 104-106, Fig. 46, 47; Capa et al. 2001c: 622.

Material examinado: Mali Rock (un ejemplar).

Distribución: Mediterráneo, Atlántico oriental, Pacífico (Japón, Islas Galápagos, Panamá).

Género Pionosyllis

Malmgren, 1867

Pionosyllis heterocirrata

(Hartmann-Schröder, 1959)

Eusyllis heterocirrata: Hartmann-Schröder, 1959: 118, Fig. 64-66; 1974: 45.

P. heterocirrata: Hartmann-Schröder 1992: 224-226, Fig. 11-15.

Material examinado: Mali Rock (tres ejemplares); Granito de Oro (siete ejemplares).

Distribución:Atlántico(IsladeAscensión), Índico (Tanzania, Sudáfrica), Pacífico (El Salvador y Panamá, primera cita).

Pionosyllis sp.

Pionosyllis sp.: Gardiner 1976: 137.

Pionosyllis cf. uraga: Day 1973: 33, Fig. 4 km. P. uraga (Non Imajima, 1966): Perkins 1981: 1108.
Material examinado: Mali Rock (un ejemplar).

Material adicional: Ejemplares del Caribe: Florida, Golfo de México, Cuba.

Discusión: Esta especie está actualmente en proceso de descripción dentro de un trabajo monográfico de revisión del género Pionosyllis (San Martín et al., en prep.).

Distribución: Atlántico (desde Carolina del Norte hasta Venezuela), Pacífico (Panamá).

\author{
Género Paraehlersia \\ San Martín, 2003 \\ Paraehlersia articulata \\ Kudenov \& Harris, 1995, n. comb
}

Pionosyllis articulata: Kudenov \& Harris 1995: 55-58, Fig. 1.20; Capa et al. 2001c: 622-623.

Material examinado: Granito de Oro (cuatro ejemplares).

Discusión: Los caracteres de esta especie coinciden con la diagnosis del género Paraehlersia de San Martín (2003), por lo que transferimos esta especie a dicho género tras comprobar la existencia de bandas ciliares y de papila subcirral, característica propia de este género. Los ejemplares coinciden con la descripción de Kudenov \& Harris (1995), aunque son de mayor tamaño. Los autores describen los cirros dorsales lisos y los dos últimos tercios de las antenas articulados y los ejemplares estudiados tienen los cirros de los primeros setígeros articulados, pero aproximadamente a partir del $4^{\circ}-5^{\circ}$ setígero se vuelven lisos y sus antenas son articuladas prácticamente desde la base. Las diferencias en el grado de articulación de los cirros dorsales anteriores están relacionadas con el tamaño de los ejemplares, siendo totalmente lisos en los más pequeños y más fuertemente articulados en los de mayor tamaño. Estas diferencias ya habían sido relacionadas por San Martín (2003) con el tamaño de los ejemplares para la especie $P$. ferrugina (Langerhans, 1881). 
Distribución: Pacífico (California, Panamá).

Género Streptosyllis

Webster \& Benedict, 1884

Streptosyllis websteri

Southern, 1914. Fig. 2

S. websteri: Southern, 1914: 26-28, pl. 2, Fig. 3S-F; Fauvel 1923: 282, Fig.107; Campoy 1982: 312; Parapar et al. 1994: 94, Fig. 1F-G; Brito et al. 2000: 607-609, Fig. 3.

Streptosyllis pettiboneae: Perkins 1981: 1143, Fig. 27 A-F, 28 A-I.

Material examinado: Granito de Oro (un ejemplar).

Descripción: Ejemplar de $2.89 \mathrm{~mm}$ de longitud, $0.19 \mathrm{~mm}$ de anchura a nivel del proventrículo (sin contabilizar apéndices) y 34 setígeros. Prostomio ovalado, dos pares de ojos dispuestos en trapecio y dos manchas oculares anteriores. Palpos pequeños no visibles dorsalmente. Una antena lateral presente, ovoide, lisa, inarticulada insertada al nivel del primer par de ojos anteriores; inserción de la central al nivel del segundo par de ojos posterior. Segmento tentacular estrecho con dos pares de cirros tentaculares inarticulados largos de más del doble de la longitud del resto de los cirros dorsales. Cirros dorsales cortos y esféricos. Cirros ventrales largos, más del doble de largos que los lóbulos parapodiales, gruesos en la base y digitiformes hacia el extremo; algunos en la región media y posterior del cuerpo, con tres o cuatro pseudoartejos con inclusiones doradas brillantes en su interior de difícil visualización. Acículas gruesas, engrosadas distalmente. En los segmentos segundo al quinto fuertemente engrosadas (Fig. 2A-E). Seda simple dorsal en todos los setígeros, en los primeros setígeros (hasta el quinto) son curvadas distalmente y con fuerte serración en el borde convexo (Fig. $2 \mathrm{G}$ ), en el resto de los segmentos sin curvatura tan marcada, con fuerte serración en su borde convexo y capuchón transparente recubriendo el extremo distal de la seda que acaba en dos pequeñas púas (Fig. 2I). En los cinco primeros setígeros hay dos sedas compuestas con artejo falcígero largo $(19 \mu \mathrm{m})$, bidentado, con filo dentado y articulación subhemigonfa; y seis sedas compuestas con el mango grueso, artejo corto $(6-12 \mu \mathrm{m})$, en algunos casos llegando a parecer bidentado, articulación engrosada, homogonfa y con algunas espinas (Fig. 2H). A partir del sexto setígero, falcígeras bidentadas, en número de seis a ocho, con articulación heterogonfa, codo anterior de la articulación provisto de un grueso diente subdistal, de artejos irregularmente dentados y en gradación dorsoventral en longitud (24 $\mu \mathrm{m}$ el más largo y $16 \mu \mathrm{m}$ el más corto, podio medio) (Fig. 2J). Tres cirros anales, dos de ellos cortos. Faringe gruesa, inerme, más corta que el proventrículo. Proventrículo ocupando 4-5 setígeros, de 45-50 filas de células musculares.

Discusión: El ejemplar coincide con la descripción y dibujos de S. pettiboneae Perkins, 1981 de Florida, que es considerado por Brito et al. (2000) como sinónima de S. websteri ya que ambas tienen acículas engrosadas y sedas compuestas modificadas en los setígeros dos a seis (aunque presenten alguna diferencia morfológica en los artejos), así como el mismo tipo de sedas compuestas a partir del setígero siete. Los tipos de S. pettiboneae han sido examinados por Brito et al. (2000) y señalan que coinciden con sus ejemplares de Canarias y que se corresponden con la descripción original de Southern (1914).

Distribución: Atlántico (desde Irlanda hasta las Islas Canarias, Golfo de México), Mediterráneo, Pacífico (Panamá, primera cita).

$$
\begin{gathered}
\text { Género Syllides } \\
\text { Örsted, } 1845 \\
\text { Syllides sp. }
\end{gathered}
$$

Material examinado: Granito de Oro (un ejemplar).

Discusión: Un único ejemplar en mal estado y de identificación dudosa. 


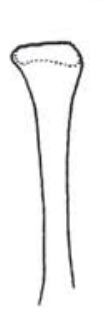

A
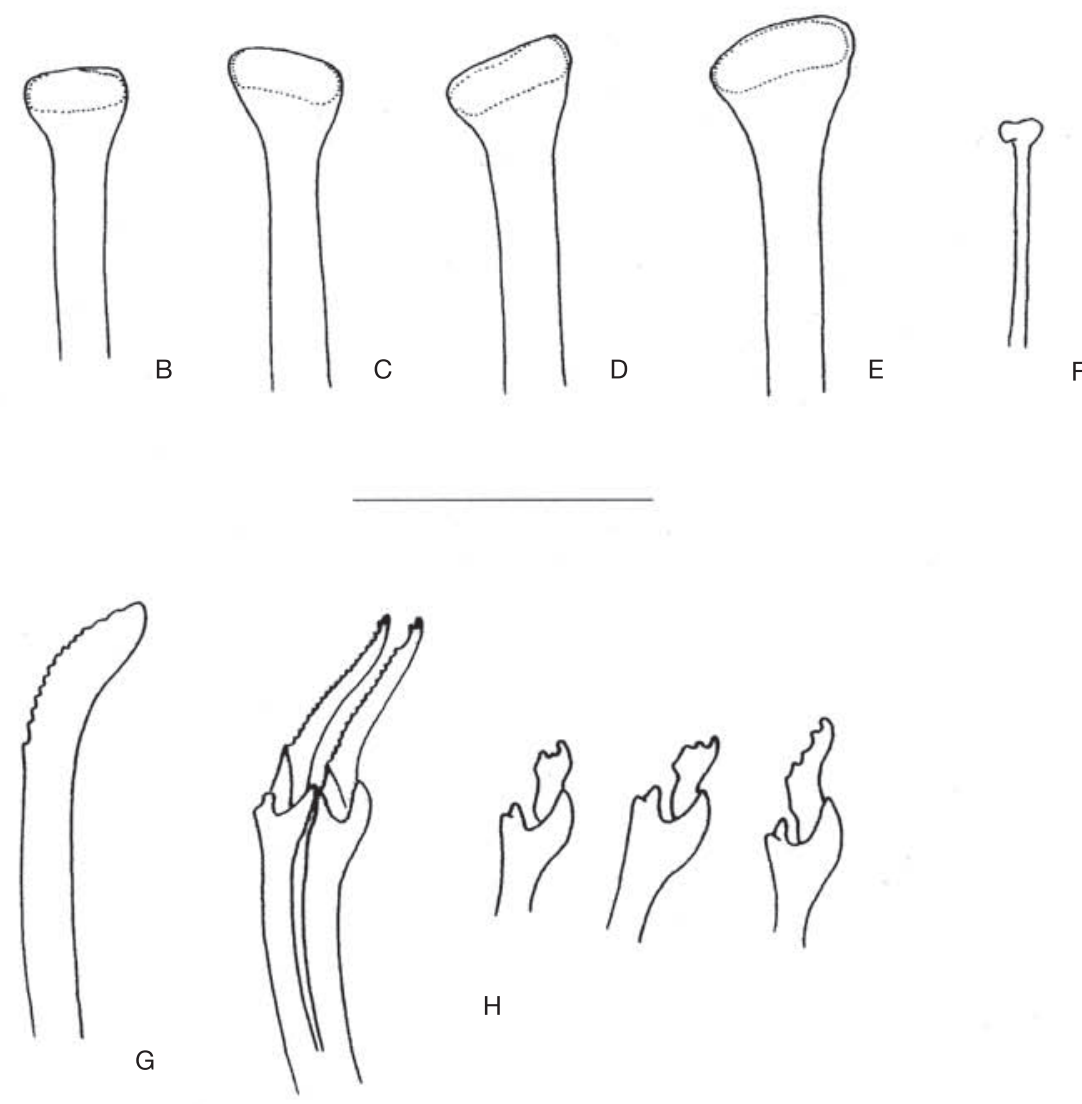

$\mathrm{H}$

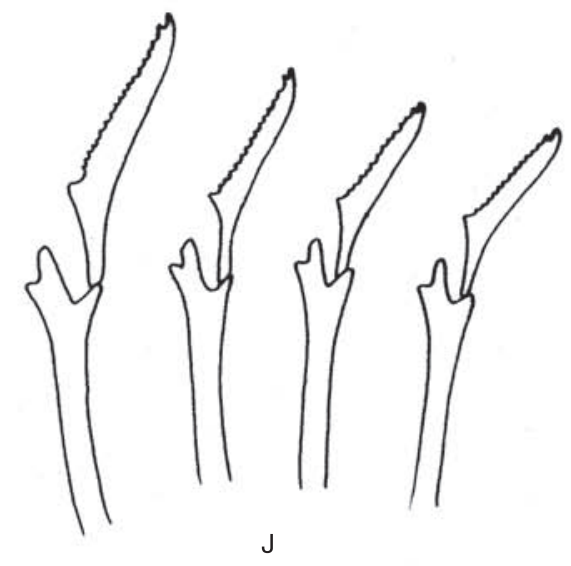

Fig. 2. Streptosyllis websteri. A. Acícula, primer setígero; B. Acícula, segundo setígero; C. Acícula, tercer setígero; D. Acícula, cuarto setígero; E. Acícula, quinto setígero; F. Acícula, a partir del sexto setígero; G. Seda simple dorsal, setígero anterior; H. Sedas compuestas, setígero anterior; I. Seda simple ventral, setígero posterior. Escala: A-J: $20 \mu \mathrm{m}$.

Fig. 2. Streptosyllis websteri. A. Acicula, first chaetiger; B. Acicula, second chaetiger; C. Acicula, third chaetiger; D. Acicula, fourth chaetiger; E. Acicula, fifth chaetiger; F. Acicula, from six chaetiger to the end of the body; G. Dorsal, simple chaeta, anterior chaetiger; H. Compound chaetae, anterior chaetiger; I. Ventral, simple chaeta, posterior chaetiger: Scale: A-J: $20 \mu \mathrm{m}$. 


\author{
Género Syllis \\ Savigny, 1818 \\ Syllis beneliahuae \\ (Campoy \& Alquezar, 1982)
}

Langerhansia beneliahui: Campoy \& Alquézar, 1982: 124-125, Fig. 3 a-p.

Syllis beneliahui: San Martín 1992: 183, Fig. $1 \mathrm{k}-\mathrm{m}$.

Typosyllis beneliahuae: Licher 1999: 47-48, Fig. 22.

S. beneliahuae: Capa et al. 2001a: 107.

Material examinado: El Gambute (nivel de bajamar) (un ejemplar).

Distribución: Mediterráneo occidental, Atlántico oriental, Caribe, Pacífico (Panamá).

\author{
Syllis botosaneanui \\ Hartmann-Schröder, 1973
}

Fig. 3-5

Typosyllis (Langerhansia) botosaneanui: Hartmann-Schröder, 1973: 90-93, Fig. 5-8; 1977: 55-56; 1980: 391; 1992: 223.

Typosyllis botosaneanui: Licher, 1999: 68-70, Fig. 31.

Syllis botosaneanui: Capa et al. 2001a: 107. Syllis garciai: San Martín, 1992: 180-181, Fig. 5 a-d. Non S. garciai Campoy, 1982.

Material examinado: Mali Rock (siete ejemplares); Granito de Oro (33 ejemplares); El Gambute (nivel intermedio de marea) (44 ejemplares); El Gambute (nivel de bajamar) (tres ejemplares).

Descripción: Uno de los ejemplares de mayor tamaño, de la muestra de Granito de Oro, tiene juveniles en su interior y se encuentra en buen estado de conservación (Fig. 3A), de $4.3 \mathrm{~mm}$ de longitud, 0.27 de anchura a nivel del proventrículo, y 46 setígeros. Cuerpo grande, largo y cilíndrico. Prostomio semicircular, con cuatro ojos dispuestos en trapecio abierto hacia la parte anterior y dos manchas oculares anteriores (justo por delante de las antenas laterales) (Fig. 3A). Tres antenas; la central más larga que las laterales, inserta entre los ojos posteriores, con 14 artejos; laterales con nueve. Palpos robustos, triangulares, de tamaño igual o mayor que el prostomio (Fig. 5A). Dos pares de cirros tentaculares, los dorsales con 12 artejos, los ventrales con nueve. Cirros dorsales del primer setígero con unos 15 artejos; los de los dos setígeros siguientes son más cortos (siete artejos); a continuación, alternando largos (810 artejos) y cortos (6-7 artejos). Dos cirros anales similares a los dorsales (7-15 artejos), y un corto apéndice anal impar inarticulado (Fig. 3B). Artejos de los apéndices con inclusiones espirales en su interior. Podios cónicos, cirros ventrales digitiformes, de longitud igual o menor que el lóbulo parapodial. Podios anteriores normalmente con dos sedas pseudoespinígeras, a veces una, presentes desde el primer setígero, con artejos de $66 \mu \mathrm{m}$ de longitud, unidentados o finamente bidentados con espinas largas y finas, y con cinco falcígeras con artejos bidentados, en gradación dorsoventral, tres más largas, de 16-18 $\mu \mathrm{m}$, con larga espinulación, que no llega a sobrepasar el nivel del diente secundario; y dos cortas, de $12-13 \mu \mathrm{m}$, con espinulación más corta (Fig. 4B, 5E). Podios medios con una pseudoespinígera similar a las anteriores pero más larga $(96 \mu \mathrm{m})$, con un diente proximal difícil de ver (Fig. 4C, 5C-D) y cuatro falcígeras dispuestas en parejas de 20 $\mu \mathrm{m}$ y 96-120 $\mu \mathrm{m}$ de longitud respectivamente, fuertemente bidentadas; los artejos de mayor longitud con espinulación desarrollada y larga (Fig. 4C). Últimos podios de disposición similar, con una pseudoespinígera unidentada, de 89 $\mu \mathrm{m}$ de longitud, y cuatro falcígeras fuertemente bidentadas, tres de ellas de 17-18 $\mu \mathrm{m}$ con espinulación más corta que las de los podios medios y anteriores y una de $14 \mu \mathrm{m}$ con espinulación también más corta y mango algo engrosado (Fig. 4D). En algunos ejemplares de mayor tamaño (16-24 mm de longitud y 113-134 setígeros), las pseudoespinígeras son considerablemente más cortas que las de los segmentos medios (aproximadamente menos de la mitad). Una acícula en cada parápodo con un ensanchamiento subdistal y una punta oblicua afilada. Una seda simple dorsal gruesa, bífida en el extremo, y muy finamente espinulada en su borde interno (Fig. 


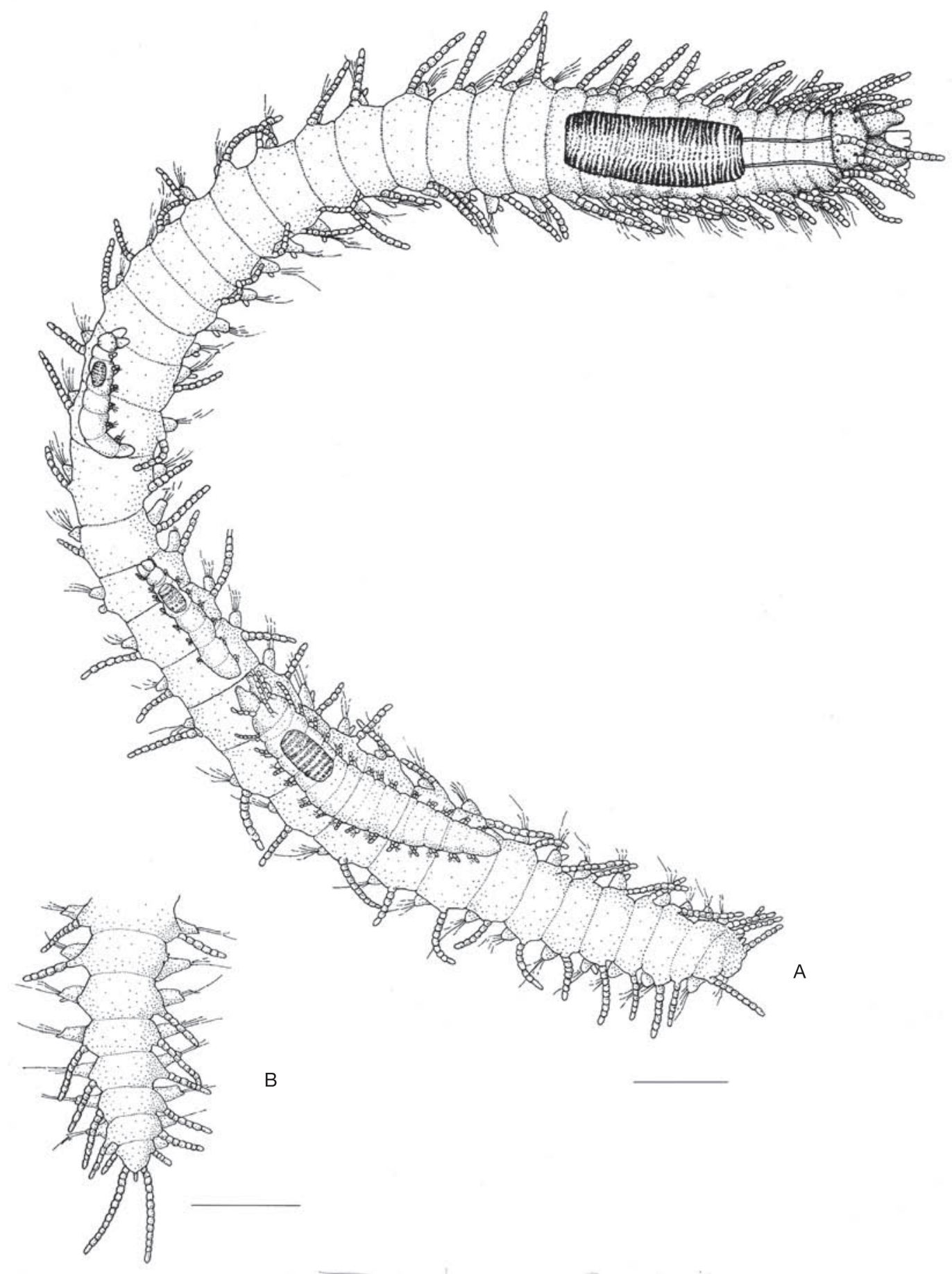

Fig. 3. Syllis botosaneanui. A. Ejemplar completo con embriones en su interior; B, Pigidio, vista dorsal. Escala A, B: 2 mm.

Fig. 3. Syllis botosaneanui. A, Complete specimen with embryos inside; B, Pygidium, dorsal view. Scale A, B: 2 mm. 

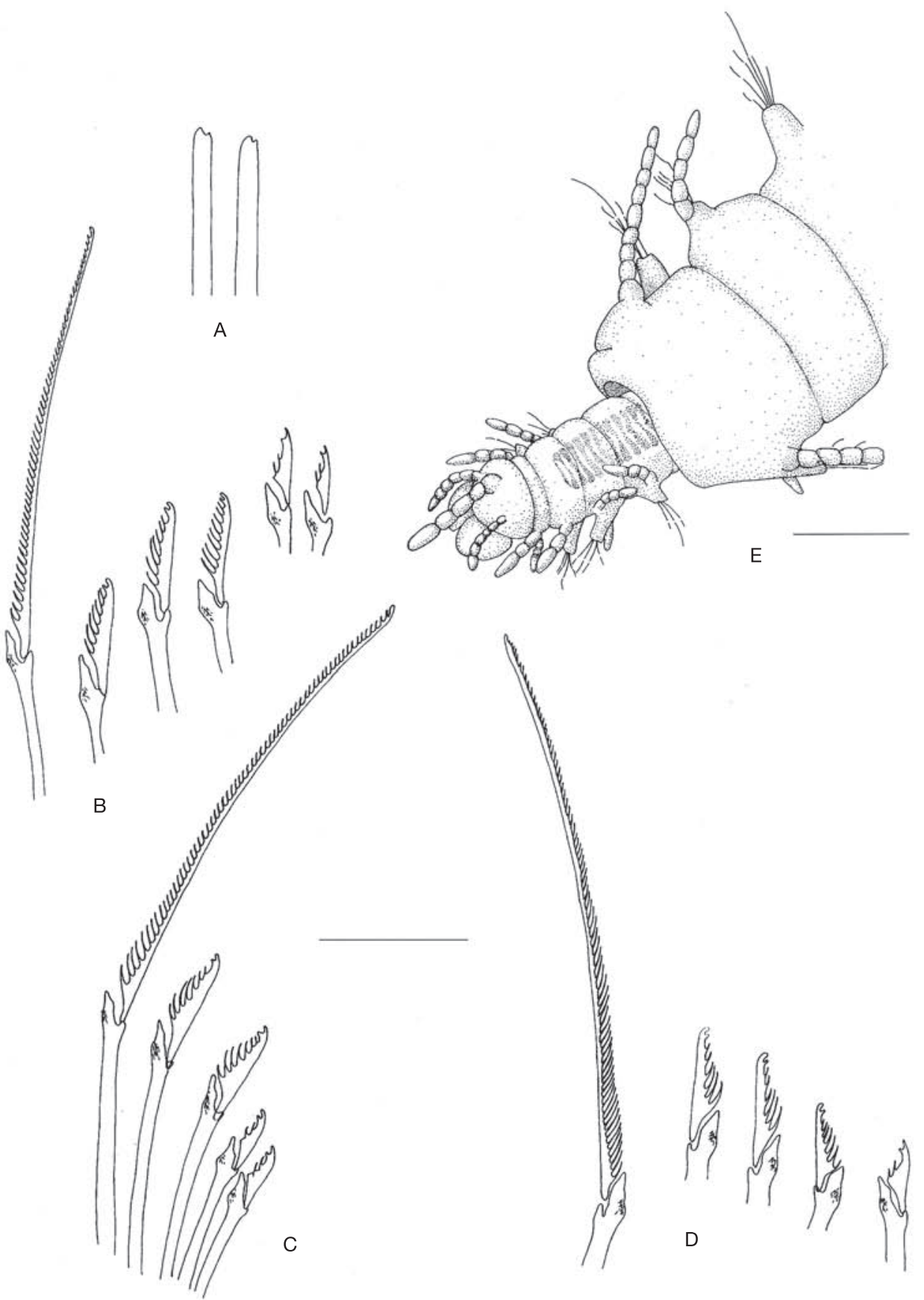

Fig. 4. Syllis botosaneanui. A, Sedas simples dorsales, setígero medio; B, Sedas compuestas, setígero anterior; C, Sedas compuestas, setígero medio; D, Sedas compuestas, setígero posterior; E, Embrión saliendo del cuerpo partido de la madre. Escala A-D: $20 \mu \mathrm{m}$; E: $97.5 \mu \mathrm{m}$.

Fig. 4. Syllis botosaneanui. A. Dorsal simple chaetae, midbody chaetiger; B. Compound chaetae, anterior chaetiger; C. Compound chaetae, midbody chaetiger; D. Compound chaetae, posterior chaetiger; E. Embryo protruding from mother's cut body. Scale A-D: $20 \mu \mathrm{m}$; E: $97.5 \mu \mathrm{m}$. 

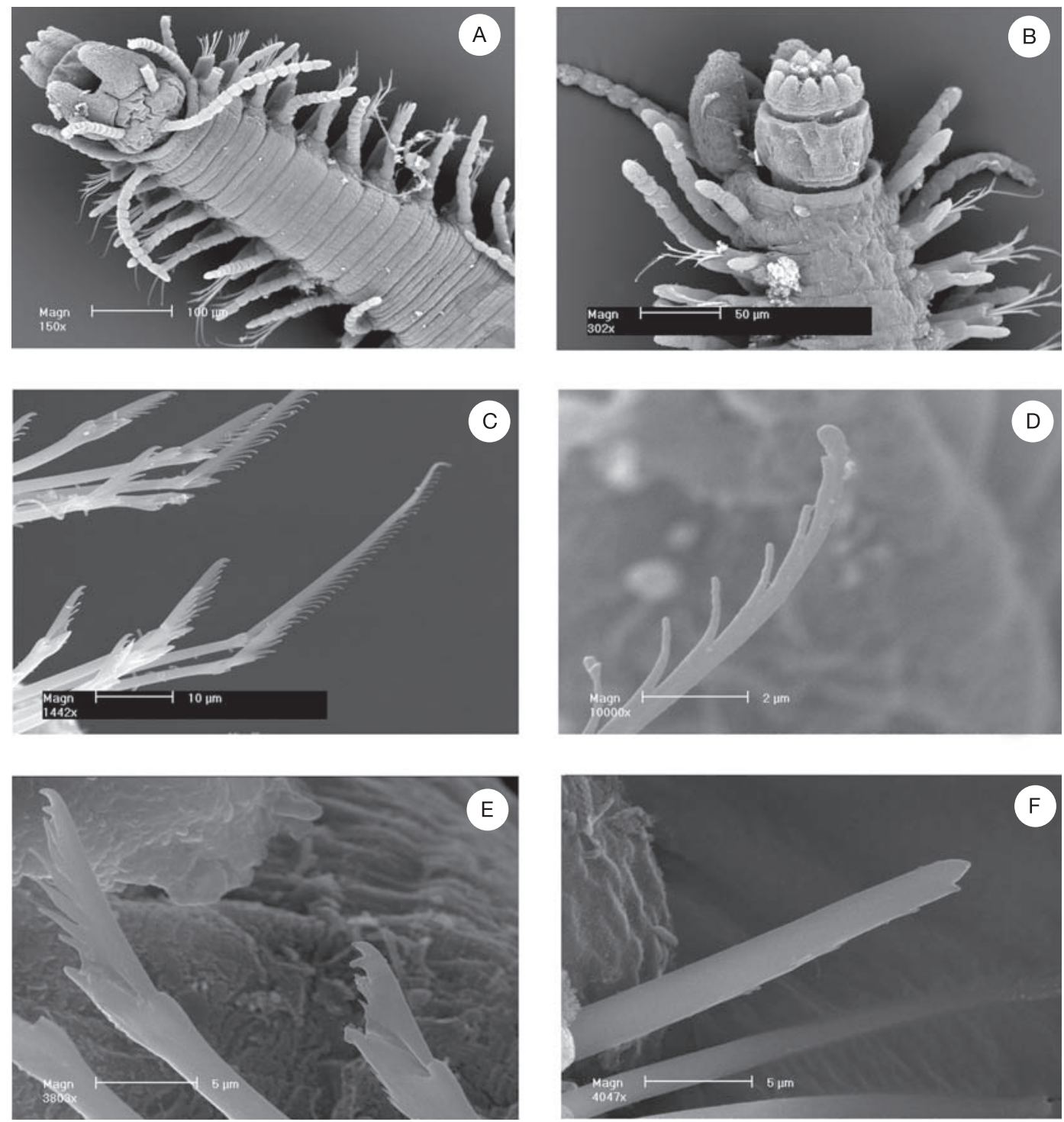

Fig. 5. Syllis sp. 1. Fotografías al microscopio electrónico de barrido (MEB). A. Parte anterior, vista dorsal; B. Parte anterior, vista ventral, probóscide evaginada; C. Sedas compuestas, setígero medio; D. Parte distal, seda compuesta pseudoespinígera, setígero medio; E. Seda falcígera inferior, setígero medio; F. Seda falcígera media, setígero medio; G. Seda simple dorsal, setígero posterior.

Fig. 5. Syllis sp. 1. Scanning electron micrographs (SEM). A. Anterior part, dorsal view; B. Anterior part, ventral view, evaginated proboscis; C. Compound chaetae, midbody chaetiger; D. Distal part, pseudospiniger chaeta, midbody chaetiger; E. Inferior falciger chaeta, midbody chaetiger; F. Middle falciger chaeta, midbody chaetiger; G. Dorsal simple chaeta, posterior chaetiger.

4A, 5F), y una seda simple ventral bidentada y finamente espinulada. Faringe cilíndrica, larga y generalmente muy oscura, con la embocadura rodeada de 10 grandes papilas (Fig. 5B) y provista de un grueso diente en su comienzo. Proventrículo de tamaño similar a la faringe con unas 33-40 filas musculares, que ocupa seis segmentos (Fig. 3A). 
Discusión: A pesar de corresponder a una misma época del año, se ha encontrado diferencias de tamaño entre los ejemplares de las distintas muestras, siendo los ejemplares de Granito de Oro los de menor longitud del cuerpo y número de setígeros. Doce ejemplares de esta muestra (de 2-4 mm de longitud), presentan en su interior embriones en diverso estado de desarrollo (Fig. 1A). Los ejemplares de Mali Rock son mucho mayores, llegando a alcanzar una longitud de 24 y 16 mm; el más pequeño tiene un juvenil en su interior. Los ejemplares de El Gambute son de tamaño intermedio (8-11 $\mathrm{mm}$ ), más delgados que los de Granito de Oro, y ninguno de ellos presenta juveniles.

De un total de 83 ejemplares revisados, 13 presentan uno o varios embriones en su cavidad celómica, en su mayoría, en la mitad posterior del cuerpo. En ninguno de los casos aparecen emergiendo del cuerpo del progenitor; uno de los ejemplares muestra en su parte final al embrión con la mitad de su cuerpo fuera debido a que probablemente se partiese justo por la zona donde se aloja la cría (Fig. 2E). Habitualmente también se observan huevos esféricos de color oscuro (uno de los ejemplares con cinco embriones y tres huevos). Normalmente, cuando aparecen varias crías en un ejemplar, éstas se encuentran en diferente estado de desarrollo. En ocasiones sólo se distinguen los palpos y los parápodos sin sedas y en otros casos se observa perfectamente su anatomía. Los embriones aparecen orientados indistintamente hacia la parte posterior o anterior del cuerpo del adulto.

S. botosaneanui y S. garciai Campoy, 1982 son dos especies muy similares, ambas citadas en Coiba por Capa et al. (2001a), que se diferencian principalmente por la longitud de la espinulación de los artejos de las sedas falcígeras, siendo más larga en S. garciai donde llega a sobrepasar el nivel del diente secundario. En los ejemplares de Coiba la espinulación de los artejos es muy larga y fina, de mayor longitud que la dibujada por Hartmann-Schröder (1973) y Licher (1999) pero no llega a sobrepasar el nivel del diente secundario. Por último, $S$. botosaneanui es una especie de fondos blandos, calificada como intersticial (HartmannSchröder 1980, Licher 1999), por lo que el hábitat coincide con el de nuestros ejemplares. Los ejemplares son determinados como $S$. botosaneanui siendo ésta la primera referencia de la especie como vivípara.

El viviparismo, como estrategia reproductora en sílidos, ha sido citada para Syllis vivipara Krohn, 1869; Syllis nepiotoca Caullery \& Mesnil, 1916; Syllis incisa Augener, 1929; Syllis parturiens (Haswell, 1920); Exogone parahomoseta mediterranea San Martín, 1984 y Exogone hebes Webster \& Benedict, 1884; y más recientemente para Dentatisyllis mangalis Russell, 1995 y Dentatisyllis mortoni Ding et al., 1998 (Krohn 1869, Goodrich 1900, Caullery y Mesnil 1916, Ben-Eliahu 1977, Pocklington y Hutcheson 1983, San Martín 1984, Russell 1995, Ding et al. 1998, Franke 1999). El viviparismo, que aparece indistintamente en diferentes subfamilias (Syllinae y Exogoninae), podría interpretarse como una adaptación al medio intersticial, más que una estrategia propia de los ciclos vitales de determinados géneros (Ding et al.1998).

Distribución: Caribe (Cuba), Índico, Atlántico occidental (Isla Ascensión), Pacífico (Panamá).

\section{Syllis glarearia}

(Westheide, 1974), n. comb.

Typosyllis glarearia Westheide, 1974: 55-58; Licher 1999: 175-177, Fig. 78.

Siguiendo los criterios de San Martín (1992, 2003), consideramos las especies descritas dentro de Typosyllis Langerhans, 1879, como pertenecientes a Syllis, por lo que esta especie constituye una nueva combinación.

Material examinado: Mali Rock (tres ejemplares); Granito de Oro (un ejemplar).

Distribución: Pacífico (Islas Galápagos y Panamá, primera cita). 
Syllis magna

(Westheide, 1974)

Ehlersia rosea magna Westheide, 1974: 41-45, Fig. 17, 18.

Typosyllis magna: Licher 1999: 49, Fig. 23.

Syllis magna: Capa et al. 2001a: 112.

Material examinado: Granito de Oro (1 ejemplar).

Distribución: Pacífico (Islas Galápagos, Panamá).

Syllis sp.

Fig. 6

Material examinado: Mali Rock (1 ejemplar: MNCN 16.01/8875)

Descripción: Único ejemplar de $14 \mathrm{~mm}$ de longitud y $0.5 \mathrm{~mm}$ de anchura a nivel del proventrículo y 64 setígeros. Cuerpo largo, cilíndrico. Prostomio subpentagonal con extremo redondeado y dos lóbulos dorsales sobre los que se encuentran los ojos, dispuestos en trapecio abierto anteriormente. Tres antenas, la central más larga que las laterales, con 17 artejos, inserta entre ambos lóbulos al nivel del par de ojos posterior; antenas laterales, con 15 artejos, insertas anteriormente, en el comienzo de los lóbulos. Dos palpos robustos, más largos que el prostomio (Fig. 6A). Dos pares de cirros tentaculares, los dorsales con 13 artejos y los ventrales con nueve. Cirros dorsales alternando irregularmente entre largos y cortos, los largos con alrededor de 30 artejos y los cortos con 1012 artejos, antenas y cirros dorsales delgados y frágiles. Cirros anales ausentes. Podios cónicos, cirros ventrales digitiformes más largos que el parápodo (Fig. 6E); cada parápodo con siete u ocho sedas compuestas heterogonfas con artejos en ligera gradación dorsoventral en longitud. Artejos cortos bidentados, diente subdistal muy pequeño, con espinas largas en la base, anchas en su comienzo, finas y dirigidas hacia arriba en el extremo distal. En podios anteriores, las tres sedas compuestas más dorsales con artejos de espinulación gruesa y patente que miden $15 \mu \mathrm{m}$ de longitud; las dos o tres sedas siguientes con artejos ligeramente menores, 11-13 $\mu \mathrm{m}$, con fina espinulación y las dos últimas sedas más ventrales con artejos de menor tamaño sin espinulación, con diente subdistal y $9 \mu \mathrm{m}$ de longitud (Fig. 6B). Forma y número de artejos similares en podios medios y posteriores, pero aumentando progresivamente de tamaño hacia la parte posterior. En los segmentos medios los tres artejos dorsales miden $16 \mu \mathrm{m}$, los dos o tres siguientes $15 \mu \mathrm{m}$ y los dos ventrales $13 \mu \mathrm{m}$; en los podios posteriores, los tres artejos dorsales miden $17 \mu \mathrm{m}$, los dos o tres medios $16 \mu \mathrm{m}$ y los dos ventrales $15 \mu \mathrm{m}$. Seda simple dorsal en podios posteriores con una pequeña escotadura en su extremo distal (Fig. 6F). Seda simple ventral ausente. Parápodos anteriores y medios con cuatro acículas delgadas con punta oblicua (Fig. 6C); los posteriores con dos acículas de punta oblicua con un engrosamiento subdistal, más gruesas que las anteriores (Fig. 6D). Faringe cilíndrica extremadamente larga, a través de 12-13 segmentos, parcialmente evertida y de color más oscuro que el resto del cuerpo, embocadura sin papilas, con un diente cónico en su comienzo. Proventrículo muy grande, unas 47-50 filas de células musculares, ocupando ocho segmentos (Fig. 6A).

Discusión: Syllis sp. se caracteriza por presentar sedas compuestas con artejos cortos, triangulares, tenuemente bidentados, provistos de espinulación larga y gruesa en la base, dirigida hacia arriba, y que se va haciendo progresivamente más corta hacia el extremo del artejo, disminuyendo el tamaño de las espinas cuanto más ventral sea la seda, llegando los artejos a ser totalmente lisos en las más ventrales, junto con una faringe muy larga y un proventrículo aproximadamente la mitad de la longitud de la faringe. Syllis glarearia (Westheide, 1974), presenta sedas con artejos más largos, con el diente proximal mayor que el distal, y con las espinas de las sedas largas en posición distal. Unas diferencias similares se pueden apreciar con respecto a Syllis lutea (Hartmann-Schröder, 1960), del mar Rojo, y S. jorgei San Martín \& López, 2000, del Mediterráneo occidental. Syllis adamanteus (Treadwell, 1914), del Pacífico Norte, Syllis 


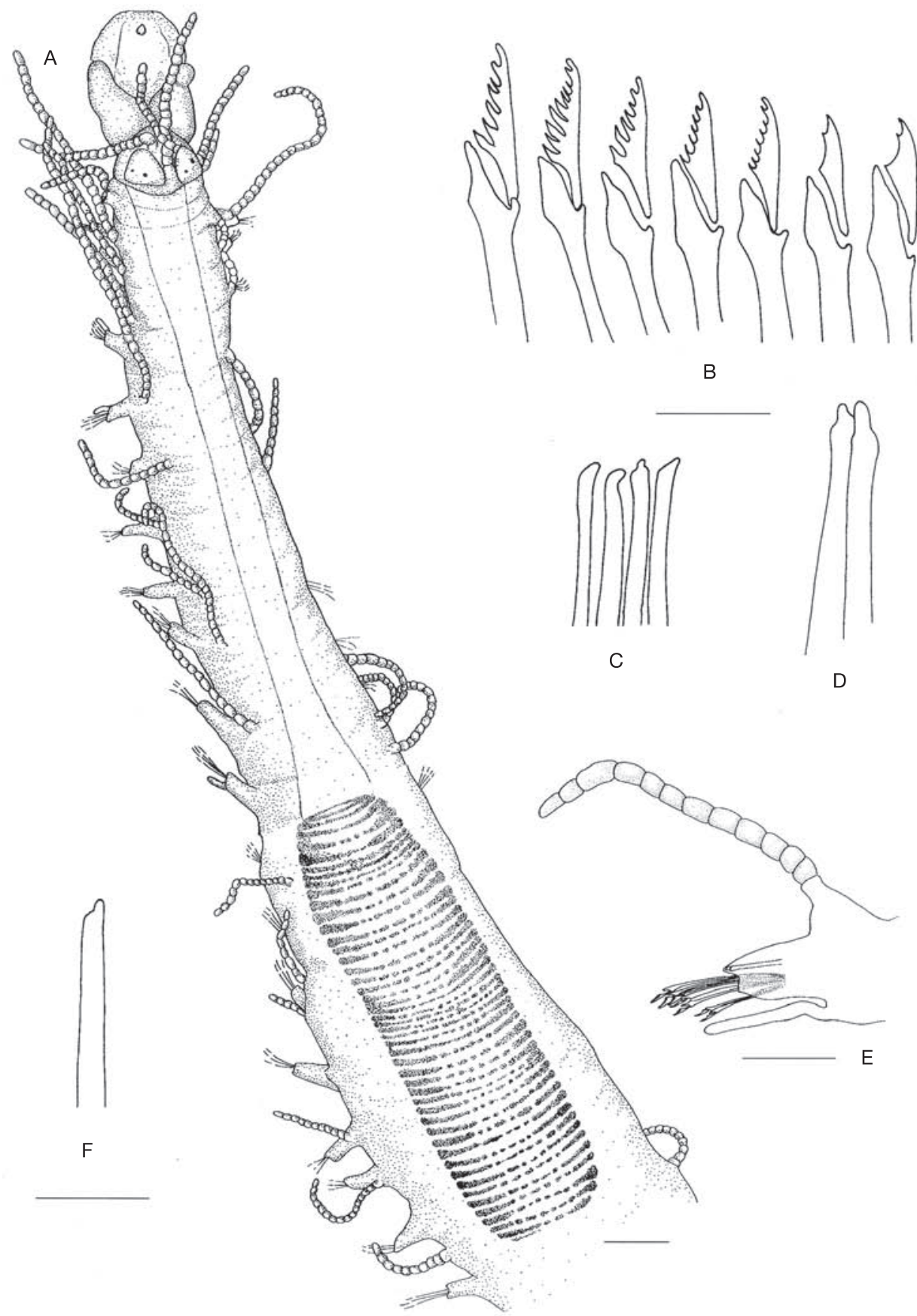

Fig. 6. Syllis sp. A. Parte anterior, vista dorsal; B. Sedas compuestas, setígero anterior; C. Acículas, setígero anterior; D. Acículas, setígero posterior; E. Parápodo derecho, segmento medio, vista anterior; F. Seda simple dorsal, setígero medio. Escala A: $97.5 \mu \mathrm{m}$; B-D, F: $10 \mu \mathrm{m}$; E: $48 \mu \mathrm{m}$.

Fig. 6. Syllis sp. A. Anterior part, dorsal view; B. Compound chaetae, anterior chaetiger; C. Aciculae, anterior chaetiger; D. Aciculae, posterior chaetiger; E. Right parapodium, midbody chaetiger, anterior view; F. Dorsal simple chaeta, midbody chaetiger. Scale A: $97.5 \mu \mathrm{m}$; B-H: $10 \mu \mathrm{m}$; I: $48 \mu \mathrm{m}$. 
dayi (Hartmann-Schröder, 1974), del Oeste de África, Syllis magdalena (Wesenberg-Lund, 1962), del Sur de Sudamérica, Syllis pectinans Haswell, 1920, de Australia, también citado en el Parque Nacional Coiba, y Syllis anoculata (Hartmann-Schröder, 1962), de Chile, son especies similares, también con sedas compuestas de artejos cortos, provistos de espinulación moderadamente larga, pero en todos los casos la sedas son unidentadas, mientras que en Syllis sp. son claramente bidentadas. Quizás, la especie más similar es Syllis licheri Ravara, San Martín \& Moreira, 2004, de las costas atlánticas de la península Ibérica, pues los artejos de las sedas son también cortos y triangulares, pero en esta especie, hay sedas bidentadas y otras unidentadas, la espinulación de los artejos es siempre mucho más corta, y una acícula muy gruesa que sobresale de los lóbulos parapodiales, lo que no sucede en Syllis sp. Ninguna de las especies similares descritas (Haswell 1920, Wesenberg-Lund 1962, Hartmann-Schröder 1973, 1974, 1991, Licher 1999, San Martín y López 2000, San Martín 2003, Ravara, 2004) presentan las características de Syllis sp. pero, al tener un solo ejemplar, preferimos no nombrarla como nueva hasta encontrar más ejemplares que corroboren las características de este espécimen.

\section{AGRADECIMIENTOS}

Agradecemos a la Agencia Española de Cooperación Internacional (AECI) la financiación parcial de las campañas llevadas a cabo en la isla de Coiba, así como al Instituto de Recursos Naturales Renovables (INRENARE) por el apoyo logístico. Agradecemos a W. Westheide la posibilidad de estudiar ejemplares de las serie tipo de E. (E.) longicornis. Apreciamos y agradecemos la inestimable ayuda de nuestros compañeros M. Capa , E. López y R. Gallego que han ayudado con sus consejos e indicaciones; además, el material de la tesis doctoral de $\mathbf{M}$. Capa ha sido utilizado para comparar ejemplares. Por último queríamos señalar que las sugerencias y correcciones aportadas por los revisores han sido de gran utilidad.

\section{RESUMEN}

Durante un estudio sobre la fauna marina del Parque Nacional de Coiba (Panamá) se identificaron 281 ejemplares de sílidos (Annelida: Polychaeta) intersticiales pertenecientes a 19 especies. Dos son nuevas citas para el Pacífico, Exogone (Exogone) arenosa Perkins, 1981 y Streptosyllis websteri Southern, 1914; cinco son nuevas citas para Panamá, E. (E.) dispar (Webster, 1879), E. (E.) longicornis Westheide, 1974, Salvatoria mediodentata (Westheide, 1974); Pionosyllis heterocirrata (HartmannSchröder, 1959) y Syllis glarearia (Westheide, 1974). Se describe un caracter no presente en la descripción original de E. Longicornis, la presencia de procesos triangulares subterminales en la primera pseudospinígera. Se describen ejemplares de la especie Syllis botosaneanui HartmannSchröder, 1973 con embriones en diverso estado de desarrollo en su interior, por lo que se trata de la primera referencia de esta especie como vivípara. Por último, se describe un ejemplar de Syllis sp., que se caracteriza fundamentalmente por la posesión de una larga faringe, dos lóbulos prostomiales dorsales y por sus sedas compuestas de artejos cortos y larga espinulación. Estas características diferencian a Syllis sp. de cualquier especie del género pero no se describe como especie nueva por disponerse únicamente de un solo ejemplar.

Palabras clave: Polychaeta, Syllidae, Coiba, Pacífico, Panamá, viviparismo.

\section{REFERENCIAS}

Banse, K. 1972. On some species of Phyllodocidae, Syllidae, Nephtyidae, Goniadidae and Spionidae (Polychaeta) from the Northeast Pacific Ocean. Pacific Sci. 26: 191-222.

Ben-Eliahu, M.N. 1977. Polychaete cryptofauna from rims of similar intertidal vermetid reefs on the Mediterranean coast of Israel and in the Gulf of Elat: Syllidae and Eusyllinae (Polychaeta: Syllidae). Israel J. Zool. 26: 1-58.

Berkeley, E. \& C. Berkeley. 1938. Notes on Polychaeta from the coast of western Canada. II. Syllidae. Pacific Biological Station, Nanaimo, B. C. Ann. \& Mag. N. Hist. ser 11, 1: 33-49.

Brito, M.C., J. Núñez \& G. San Martín. 2000. The genus Streptosyllis Webster and Benedict, 1884 (Polychaeta: 
Syllidae: Eusyllinae) from the Canary Islands, with description of a new species. Bull. Mar. Sci. 67: 603-615.

Campoy, A. 1982. Fauna de España. Fauna de anélidos poliquetos de la Península Ibérica. Universidad de Navarra. Ser. Biol. 7: 1-781

Campoy, A. \& E. Alquézar. 1982. Anélidos poliquetos de las formaciones de Dendropoma petraeum (Monterosato) de las costas del sureste de España. Actas II Simp. Est. Bentos Mar. 3: 121-137.

Capa, M., G. San Martín \& E. López. 2001a. Syllinae (Syllidae: Polychaeta) del Parque Nacional de Coiba (Panamá). Rev. Biol. Trop. 48: 101-113.

Capa, M., G. San Martín \& E. López. 2001b. Description of a new species of Parasphaerosyllis (Polychaeta: Syllidae: Syllinae). Proc. Biol. Soc. Wash. 114: 280-284.

Capa, M., G. San Martín \& E. López. 2001c. Autolitynae, Eusyllinae y Exogoninae (Syllidae: Polychaeta) con comentarios sobre la ecología y biogeografía de la familia Syllidae del Parque Nacional de Coiba, Panamá. Rev. Biol. Trop. 49: 621-628.

Caullery, M. \& F. Mesnil. 1916. Viviparité et parthenogenèse chez les Annélides Polychètes: un nouveau Syllidien vivipare. C. R. Acad. Sci. Paris 163: 576579.

Day, J.H. 1973. New Polychaeta from Beaufort, with a key to all species recorded from North Carolina. In NOAA Tech. Rep. NMFS CIRC 375, Seattle, Wash., EEUU. 140 p.

Díaz-Castañeda, V. \& G. San Martín. 2001. Syllidae (Polychaeta) from San Quintín Bay, Baja California, Mexico, with the description of a new genus. Proc. Biol. Soc. Wash. 114 : 708-719.

Ding, Z., F. Licher \& W. Westheide. 1998. New and newly assigned species of the genus Dentatisyllis (Polychaeta: Syllidae: Syllinae), with comments on the reproduction, together with a key and synoptic table of all species of the genus. Sarsia 83: 29-43.

Fauvel, P. 1923. Faune de France. Vol. 5: Polychètes Errantes. Libraire de la Faculte des Sciences Paul Lechevalier, París, Francia. 488 p.

Franke, H.D. 1999. Reproduction of the Syllidae (Annelida: Polychaeta). Hydrobiologia 402: 39-55.

Gardiner, S.L. 1976. Errant polychaete annelids from North Carolina. J. Elisha Mitchell Sci. Soc. 91: 77-220.

Goodrich, E.S. 1900. Observations on Syllis vivipara Krohn. J. Linn. Soc. Zool. 28: 105-108.
Hartmann-Schröder, G. 1959. Zur Ökologie der Polychaeten des Magrove-Estero-Gebietes von El Salvador. Beitr. neotrop. Fauna 1: 70-183.

Hartmann-Schröder, G. 1960. Polychaeten aus dem Roten Meer. Kiel. Meeresforsch. 16: 69-125.

Hartmann-Schröder, G. 1973. Die Polychaeta der Biospeologischen Expedition nach Kuba 1969. Résul. Exped. Bioespéol. Cubano-roum 1: 89-98.

Hartmann-Schöder, G. 1974. Zur Kenntnis des Eulitorals der afrikanischen Westküste zwischen Angola und Kap der Gunten Hoffnung und der afrikanischen Ostküste von Südafrika und Mocambique unter besonderer Berücksichtigung der Polychaeten und Ostracoden. Teil 2. Die Polychaeten des Untercungsgebietes. Mitt. hamb. Zool. Mus. Inst. 69: 95-228.

Hartmann-Schöder, G. 1977. Die Polychaeten der Kubanisch-Rumänischen Biospeologischen Expedition nach Kuba 1973. Rés. Exp. Biospéol. Cub.-roum. 2: 51-63.

Hartmann-Schöder, G. 1980. IX. Die Polychaeten der Amsterdam-Expedionen nach Westindien. In Amsterdam expeditions to the West Indian Islands. Bijdr. Dierk., 50: 387-401.

Hartmann-Schröder, G. 1991. Zur Kenntnis des Eulitorals der australischen Küsten unter besonderer Berücksichtigung der Polychaeten und Ostracoden. Teil 16. Polychaeten der subtropisch-tropischen bis tropischen Ostküste Australiens zwischen Lake Maclean (New South Wales) und Gladstone (Queensland) sowie von Heron Island (Großes Barries Riff). Mitt. Hamb. Zool. Mus. Inst. 88: 17-71.

Hartmann-Schröder, G. 1992. Die Polychaeten der Amsterdam-Expedition nach der Insel Ascension (Zentral-Atlantik). Bijdragen tot de Dierkunde 61: 219-235.

Haswell, M.A. 1920. Australian Syllidae, Eusyllidae and Autolytidae. Proc. Linn. Soc. N. S. W. 45: 90-112.

Imajima, M. \& O. Hartman. 1964. The polychaetous annelids of Japan. Allan Hancok Fdn. Occ. Pap. 26: 1-452.

Imajima, M. 1966. The Syllidae (Polychaetous Annelids) from Japan (III). Eusyllinae. Publ. Seto Mar. Biol. Lab. 14: 85-116.

Krohn, A. 1869. Ueber eine lebendiggebärende Syllis Art. Arch. Naturgesch. 35: 197-200.

Kudenov, J.D \& L. Harris. 1995. Family Syllidae Grube, 1850, p. 1-97. In J.A. Blake, B. Hilbig \& P.H. Scott (eds.). Taxonomic Atlas of the Benthic Fauna of the Santa María Basin and Western Santa Bárbara 
Channel. Vol 5. The Annelida, Part 2. Santa Barbara Museum Nat. Hist., Santa Barbara, California, EEUU.

Licher, F. 1999. Revision der Gattung Typosyllis Langerhans, 1879 (Polychaeta: Syllidae). Morphologie, Taxonomie und Phylogenie. Abh. Senckenberg. Naturforsch. Ges. 551: 1-336.

López, E., G. San Martín, P. Cladera \& M. Capa. 1997. La fauna de anélidos poliquetos del Parque Nacional de Coiba (Panamá), p. 57-73. In S. Castroviejo (ed.). Flora y Fauna del Parque Nacional de Coiba (Panamá). AECI. Madrid, España.

López, E., P. Cladera, G. San Martín, A. Laborda \& M.T. Aguado. 2002. Polychaete assemblages inhabitting intertidal soft bottoms associates to mangrove systems from Coiba National Park (East Pacific, Panamá). Wet. Ecol. Manag. 10: 233-242.

Núñez, J., G. San Martín \& M.C. Brito. 1992. Exogoninae (Polychaeta: Syllidae) from the Canary Islands. Sci. Mar. 56: 43-52.

Parapar, J., G. San Martín, C. Besteiro \& V. Urgorri. 1994. Aspectos sistemáticos y ecológicos de las subfamilias Eusyllinae y Exogoninae (Polychaeta, Syllidae) en la Ría de Ferrol (Galicia, NO España). Bol. R. Soc. Esp. Hist. Nat. (Sec. Biol.) 91: 91-101.

Perkins, T.H. 1981. Syllidae (Polychaeta), principally from Florida, with description of a new genus and twenty-one new species. Proc. Biol. Soc. Wash. 93: 1080-1172.

Pettibone, M.H. 1963. Marine polychaete worms of the New England region. Part 1. Families Aphroditidae through Trochochaetidae. Bull. U. S. Nat. Mus. 227: $1-356$.

Pocklington, P. \& M.S. Hutcheson. 1983. New record of viviparity for the dominant benthic invertebrate Exogone hebes (Polychaeta: Syllidae) from the Grand Banks of Newfoundland. Mar. Ecol. 11: 239-244.

Ravara, A., G. San Martín \& M.H. Moreira. 2004. Syllidae (Annelida, Polychaeta) from the continental shelf off Aveiro (NW Portugal) with the description of a new species, Syllis licheri. Senckenberg. Biol. 84:1-11.

Rioja, E. 1941. Estudios anelidológicos III. Datos para el conocimiento de la fauna de poliquetos de la costa del Pacífico de México. An. Inst. Biol. Mexicano 18: 197-220.

Russell, D.E. 1991. Exogoninae (Polychaeta: Syllidae) from the Belizean barrier reef with a key to species of Sphaerosyllis. J. Nat. Hist. 25: 49-74.
Russell, D.E. 1995. Description of a new viviparous species of Dentatisyllis (Polychaeta: Syllidae) from Belize with an assessment of growth and variation, and emendation of the genus. Proc Biol. Soc. Wash. 108: 568-576.

San Martín, G. 1984. Estudio biogeográfico, faunístico y sistemático de los poliquetos de la familia sílidos (Syllidae: Polychaeta) en Baleares. Ph.D. Thesis, Univ. Complutense Madrid, España. 529 p.

San Martín, G. 1991. Grubeosyllis and Exogone (Exogoninae, Syllidae, Polychaeta) from Cuba, the Golf of Mexico, Florida and Puerto Rico, with a revision of Exogone. Bull. Mar. Sci. 49: 751-740.

San Martín, G. 1992. Syllis Savigny in Lamark, 1818 (Polychaeta: Syllidae: Syllinae) from Cuba, the Gulf of Mexico, Florida and North Carolina, with a revision of several species described by Verrill. Bull. Mar. Sci. 51: 167-196.

San Martín, G. 2000. El Parque Nacional de Coiba (Panamá). Estudio del bentos marino. Bol. R. Soc. Esp. Hist. Nat. (actas) 97: 17-23.

San Martín, G. 2001. El medio marino en el Parque Nacional de Coiba (Panamá). Quercus Octubre 188: 33-35.

San Martín, G. 2003. Annelida, Polychaeta II: Syllidae In M.A. Ramos et al. (eds.). Fauna Ibérica, Vol. 21. Museo Nacional de Ciencias Naturales, CSIC, Madrid, España.

San Martín, G. 2005. Exogoninae (Polychaeta, Syllidae) from Australia (with the description of a new genus and twenty-two new species). Rec. Aus. Mus. 57: 39-152

San Martín, G., P. Cladera, E. López, A. Laborda, F. García \& M.S. Redondo. 1997a. Las Aguas Marinas, p. 45-62. In M. Velayos, C. Monge, F. Posse \& S. Castroviejo (eds.). Guía de Campo del Parque Nacional de Coiba (Panamá). AECI, Madrid, España.

San Martín, G., E. López, M.S. Redondo, M. Capa, P. Cladera \& A. Laborda. 1997b. El bentos marino del Parque Nacional de Coiba (Panamá), p. 33-55. In S. Castroviejo (ed.). Flora y Fauna del Parque Nacional de Coiba (Panamá). AECI, Madrid, España.

San Martín, G. \& E. López. 2000. Three new species of Syllis (Syllidae: Polychaeta) from Iberian coast. Cah. Biol. Mar. 41: 425-433.

Southern, R. 1914. Clare Island Survey. Archiannelida and Polychaeta. Proc. R. I. Acad. 31: 1-160. 
Uebelacker, J.M. 1984. Family Syllidae. Chapter 30, p. 30-30. In J.M. Uebelacker \& P.G. Johnson (eds.). Taxonomic guide to the polychaetes of the northern Gulf of Mexico, Vol. IV. Barry Vittor, Alabama, EEUU.

Wesenberg-Lund, E. 1962. Polychaeta Errantia. In Reports of the Lund University Chile Expedition 1948-49. Lunds Univ. Arsskr, n.f., avd. 2. 57: 1-137.
Westheide, W. 1974. Interstitial Fauna von Galapagos XI. Pisionidae, Hesionidae, Pilargide, Syllidae (Polychaeta). Mikr. des Meere 44: 1-146.

Zottoli, R. \& C.D. Long. 2000. Exogone breviantennata Hartmann-Schröder, 1959 (characters emended) (Annelida: Polychaeta: Syllidae), a new record for the Bahamas with a key to selected Exogone species. Proc. Biol. Soc. Wash. 113: 500-513. 
